Original paper

\title{
Performance and limitations of positron emission tomography (PET) scanners for imaging very low activity sources
}

\author{
Melissa I. Freedenberg a , Ramsey D. Badawi ${ }^{\text {a,b }}$, Alice F. Tarantal ${ }^{\text {c,d,e }}$, Simon R. Cherry ${ }^{\text {a,b,e, } *}$ \\ ${ }^{a}$ Department of Biomedical Engineering, University of California, Davis, CA 95616, USA \\ ${ }^{\mathrm{b}}$ Department of Radiology, University of California, Davis, CA 95616, USA \\ ${ }^{\mathrm{C}}$ Department of Pediatrics, University of California, Davis, CA 95616, USA \\ ${ }^{\mathrm{d}}$ Department of Cell Biology and Human Anatomy, University of California, Davis, CA 95616, USA \\ e California National Primate Research Center, University of California, Davis, CA 95616, USA
}

\section{A R T I C L E I N F O}

\section{Article history:}

Received 10 December 2012

Received in revised form

21 March 2013

Accepted 19 April 2013

Available online 14 May 2013

\section{Keywords:}

Positron emission tomography scanners

Low activity

Performance

\begin{abstract}
A B S T R A C T
Emerging applications for positron emission tomography (PET) may require the ability to image very low activity source distributions in the body. The performance of clinical PET scanners in the regime where activity in the field of view is $<1 \mathrm{MBq}$ has not previously been explored. In this study, we compared the counting rate performance of two clinical PET/CT scanners, the Siemens Biograph Reveal 16 scanner which is based on lutetium oxyorthosilicate (LSO) detectors and the GE Discovery-ST scanner which is based on bismuth germanate (BGO) detectors using a modified National Electrical Manufacturers Association (NEMA) NU 2-2007 protocol. Across the activity range studied $(2-100 \mathrm{kBq} / \mathrm{mL}$ in a $5.5 \mathrm{~mL}$ line source in the NEMA scatter phantom), the BGO-based scanner significantly outperformed the LSO-based scanner. This was largely due to the effect of background counts emanating from naturally occurring but radioactive ${ }^{176} \mathrm{Lu}$ within the LSO detector material, which dominates the observed counting rate at the lowest activities. Increasing the lower energy threshold from $350 \mathrm{keV}$ to $425 \mathrm{keV}$ in an attempt to reduce this background did not significantly improve the measured NECR performance. The measured singles rate due to ${ }^{176} \mathrm{Lu}$ emissions within the scanner energy window was also found to be dependent on temperature, and to be affected by the operation of the CT component, making approaches to correct or compensate for the background more challenging. We conclude that for PET studies in a very low activity range, BGO-based scanners are likely to have better performance because of the lack of significant background.
\end{abstract}

( 2013 Associazione Italiana di Fisica Medica. Published by Elsevier Ltd. All rights reserved.

\section{Introduction}

Clinical positron emission tomography (PET) scanners have been designed and optimized for imaging injected radiotracer doses in the range of $\sim 50-750 \mathrm{MBq}$. However, a number of emerging applications for in vivo imaging may utilize orders of magnitude lower injected activities. For example, radiolabeling of cells and drug carriers such as nanoparticles can be limited by radiotoxicity and/or the number of cells/particles, with injected doses as low as $0.5-5 \mathrm{MBq}$. Under these circumstances, sources of noise and background that are insignificant at the counting rates generated by conventional injected doses may become limiting for signal detection and quantification.

\footnotetext{
* Corresponding author. Department of Biomedical Engineering, 1 Shields Avenue, University of California, Davis, Davis, CA 95616. Tel.: +1530 7549419.

E-mail address: srcherry@ucdavis.edu (S.R. Cherry).
}

In this study we assessed the counting rate performance of two clinical PET scanners, based on different detector materials, as the activity in the field of view decays to near background levels. One scanner (Siemens Biograph Reveal 16) utilizes detectors based on the scintillator lutetium oxyorthosilicate $\left(\mathrm{LSO}, \mathrm{Lu}_{2} \mathrm{SiO}_{5}: \mathrm{Ce}\right)$, while the second scanner (GE Discovery-ST) uses the scintillator bismuth germanate ( $\mathrm{BGO}, \mathrm{Bi}_{4} \mathrm{Ge}_{3} \mathrm{O}_{12}$ ). The goal of this study was to investigate the performance of these two widely used clinical scanners in the poorly studied regime in which very low activity radiotracer concentrations are contained in the imaging field of view, and determine if there are significant differences between them. A secondary goal was to better understand factors that influence performance at low activity concentrations. Because LSO scintillator contains a small amount of naturally occurring radioactive ${ }^{176} \mathrm{Lu}$, which produces a non-negligible signal background, we hypothesized that the two clinical scanners may behave very differently at low activity concentrations. 
To evaluate the Biograph and Discovery-ST counting rate performance, we used the widely adopted National Electrical Manufacturers Association (NEMA) protocols for decay series counting rate measurements [1], which were adapted for low-activity studies using previously published recommendations [2]. Based on our findings with these measurements, we supplemented our assessment by evaluating the relationship between temperature and scanner counting rate.

While counting rate performance has previously been measured for both the LSO-based Siemens Biograph and BGO-based Discovery-ST scanners, these studies focused on typical clinical radioactivity concentrations [3-7]. Although the magnitude of the signal background resulting from natural ${ }^{176} \mathrm{Lu}$ in LSO-based scanners has been characterized [8-10], there is only one published study to our knowledge that examines the effect of ${ }^{176} \mathrm{Lu}$ background on measurements of weak sources in the field of view of a preclinical scanner [11]. We know of no previously published studies that have compared the performance of clinical scanners based upon the most common PET detector materials LSO and BGO in this lowactivity range. Extrapolation of the results from a preclinical scanner is not straightforward, because of the 20-40-fold lower volume of scintillator material, and differences in energy resolution and typical energy windows employed. The major contribution of this work therefore is to compare two clinical scanners based on different detector materials to determine the effect of radioactive background on low-count measurements using standard assessment tools based on the NEMA protocol. The results from these studies should be of interest to those wishing to image very low activity concentrations on clinical PET/CT scanner platforms.

\section{Materials and methods}

\section{PET scanners}

The characteristics of the PET scanners used to obtain measurements are summarized in Table 1 . The two clinical whole-body PET/CT scanners evaluated, the GE Discovery-ST and the Siemens Biograph Reveal 16, were in routine clinical use at the time the measurements were made. The Biograph scanner utilizes modular detectors based on LSO scintillator coupled to photomultiplier tubes (PMTs). LSO contains a small amount of naturally occurring radioactive ${ }^{176}$ Lu that has an activity of approximately $330 \mathrm{~Bq}$ per cc of LSO material. The Discovery-ST scanner uses modular BGO-based scintillation detectors; BGO has negligible natural radioactivity and typically has no significant radioactive contaminants. Other than

Table 1

Summary of relevant characteristics of clinical PET/CT scanners used in this study.

\begin{tabular}{lll}
\hline Scanner & $\begin{array}{l}\text { Siemens Biograph } \\
\text { Reveal 16 }\end{array}$ & GE Discovery-ST \\
& LSO & BGO \\
\hline Scintillator & $6.45 \times 6.45 \times 25$ & $6.3 \times 6.3 \times 30$ \\
Crystal size $\left(\mathrm{mm}^{3}\right)$ & 162 & 157 \\
Axial field-of-view $(\mathrm{mm})$ & 830 & 886 \\
Ring diameter (mm) & 24 & 24 \\
Crystal rings & 384 & 420 \\
Crystals per ring & $8 \times 8$ & $6 \times 6$ \\
Crystal block size & 0.5 & 5.8 \\
Coincidence timing & 4.5 & 11.7 \\
$\quad$ resolution (ns) & $<19$ & 17 \\
Default timing window (ns) & $6.3 \mathrm{~mm}(10 \mathrm{~mm})$ & $6.28 \mathrm{~mm}(10 \mathrm{~mm})$ \\
Energy resolution (\%) & & \\
Transaxial spatial resolution & $5.7 \mathrm{cps} / \mathrm{kBq}$ & $9.26 \mathrm{cps} / \mathrm{kBq}$ \\
$\quad($ Offset) & $(400-650 \mathrm{keV})$ & $(375-650 \mathrm{keV})$ \\
Sensitivity (energy window) & {$[7]$} & {$[3,6]$} \\
References & & \\
\hline
\end{tabular}

the different scintillation materials used, the two scanners studied are very similar in terms of overall detector and scanner geometry.

For whole-body clinical PET scanners there has been a trend towards LSO (or closely related LYSO) based scanners because LSO has higher light output and a fast signal decay time which leads to better timing resolution and reduces dead time for event detection at higher counting rates compared with BGO-based scanners [12]. However, BGO has a somewhat higher linear attenuation coefficient at $511 \mathrm{keV}\left(0.95 \mathrm{~cm}^{-1}\right.$ versus $0.87 \mathrm{~cm}^{-1}$ for LSO), thus it has a higher probability of detecting an annihilation photon emitted from the subject. This, combined with the lack of background due to intrinsic radioactivity, suggests that BGO may be a better choice when imaging at low activities.

\section{Phantom}

A standard cylindrical NEMA NU 2-2007 scatter phantom (Data Spectrum Corp, Hillsborough, NC) was used for all counting rate measurements. The phantom is a solid right circular cylinder with a diameter of $20.3 \mathrm{~cm}$ and a length of $70 \mathrm{~cm}$ made from polyethylene. The phantom has a $6.4 \mathrm{~mm}$ diameter hole along its axis, located at an axial offset of $4.5 \mathrm{~cm}$ from the center of the cylinder, which holds $3.2 \mathrm{~mm}$ internal diameter tubing that is filled with a known quantity of radioactivity.

\section{Sources}

${ }^{18} \mathrm{~F}$ (in the form of ${ }^{18} \mathrm{~F}$-fluorodeoxyglucose, FDG) was obtained from PETNET (Sacramento, CA) for studies on the Biograph scanner and IBA Molecular (Gilroy, CA) for studies on the Discovery-ST scanner. Supplied ${ }^{18} \mathrm{~F}$ activities were verified using clinical dose calibrators at each site. ${ }^{18}$ FDG solutions were well-mixed, and dilutions were performed by weight and volume. To enable comparisons between the two scanners, a cross-calibration between dose calibrators at each site was performed using a $7.4 \mathrm{MBq}{ }^{137} \mathrm{Cs}$ source. A volume of $5.5 \mathrm{~mL}$ of ${ }^{18} \mathrm{FDG}$ solution was used to fill the tubing along the axial extent of the scatter phantom. Measurements were made starting with an initial activity in the line source of $\sim 600 \mathrm{kBq}$, and continued until the source activity had decayed to $\sim 2 \mathrm{kBq}$.

\section{Counting rate measurements}

Counting rate measurements followed a modified version of the NEMA NU-2-2007 counting rate procedure [1,2]. This included both a high-statistics NEMA scatter fraction measurement and the standard NEMA counting rate analysis with provisions for scanners with intrinsic radioactivity.

For the Biograph scanner, low-activity decay series were acquired for line source activities ranging from $106 \mathrm{kBq} / \mathrm{ml}$ to $2 \mathrm{kBq} /$ $\mathrm{ml}$ with the 6 ns default timing window and a 375-650 keV energy window. To study the effect of the energy window on low-activity counting rate performance, data were also acquired using a lower energy threshold of $350 \mathrm{keV}$ and $425 \mathrm{keV}$ over a more limited activity range of $13 \mathrm{kBq} / \mathrm{ml}$ to $2 \mathrm{kBq} / \mathrm{ml}$. Additional scans were acquired to measure the scatter fraction for each energy window. The activity in the line source was chosen to minimize the randoms-totrues ratio in accordance with the recommendations of Watson et al. [2].

The decay series on the Discovery-ST scanner was acquired as line source activity decayed from $233 \mathrm{kBq} / \mathrm{mL}$ to $0.1 \mathrm{kBq} / \mathrm{mL}$, using the default $11.7 \mathrm{~ns}$ timing window and a 375-650 keV energy window. Previously published Discovery-ST scatter fraction values using the NEMA NU-2-2007 protocol were used in the analysis [3]. On both scanners, prompt and random coincidences were acquired 
simultaneously, with random coincidences acquired by using a delayed coincidence window.

On each scanner, a background (phantom in scanner, but no source) counting rate acquisition was performed per recommendations by Watson et al. [2]. A 12-h background dataset was acquired for each lower energy threshold setting on the Biograph scanner, and a 24-h background data set was acquired on the Discovery scanner.

Prior to each PET acquisition, an X-ray computed tomography (CT) scan was acquired for phantom positioning and to verify the absence of air bubbles in the phantom line source. For the Biograph scanner, a three bed position CT acquisition was performed with an effective tube output of $200 \mathrm{mAs}$ and a tube voltage of $120 \mathrm{kVp}$. For the Discovery-ST scanner, a whole body CT acquisition was performed with a of tube output of $392 \mathrm{mAs}$ and a tube voltage of $120 \mathrm{kVp}$.

Data were histogrammed into single-slice rebinned sinograms with ring difference 17 and span 7 using a custom Matlab script for the Discovery-ST data and scanner software for the Biograph data. All segments were subsequently combined to form a single sinogram containing all lines of response (LORs). This is the same sinogram configuration used in a previously published NEMA count rate study by Martinez et al. [5].

\section{Counting rate analysis}

Analysis of the counting rate data from each decay series was performed according to the methodology described in the NEMA NU 2-2007 standard [1], with provisions for scanners with intrinsic radioactivity [2]. Sinograms from the first frame of each emission scan were cropped and shifted such that the maximum value in each projection angle was aligned with the central pixel of the sinogram. The mapping between the original and shifted sinogram was stored and applied to each successive frame such that proper source positioning was preserved as activity decayed to background levels. The same mapping was also applied to random and background sinograms. Each shifted sinogram slice was then summed across all projection angles to form a one-dimensional profile. From the measured prompt, random and intrinsic background counting rates, as well as the scatter fraction, the noise equivalent counting rate was calculated as a function of the line source activity concentration for the two scanners.

\section{Sinogram contrast-to-noise ratio (CNR) analysis}

Biograph and Discovery-ST decay series sinogram planes were summed to yield a single transaxial sinogram for each acquisition frame. The first four frames of each acquisition were summed to form a high-statistics sinogram, and a source region-of-interest (ROI) was defined by applying a threshold to this sinogram. A background ROI was defined for the Biograph scanner to be $38.7 \mathrm{~mm}$ (6 sinogram bins wide) on each side of the source ROI, and offset from the source by $25.8 \mathrm{~mm}$ in each direction. For the Discovery-ST scanner, the background ROI was defined to be $37.8 \mathrm{~mm}$ wide (6 sinogram bins wide) on each side of the source ROI, and offset from the source by $25.2 \mathrm{~mm}$ in each direction. The slight difference in dimensions between the two scanners reflects differences in the radial sampling of the sinogram bins. The standard deviation within the background ROIs was taken as a measure of the noise. The CNR was defined as:

$$
\begin{aligned}
& \mathrm{CNR}=\left\{\left(\text { mean }\left(\mathrm{ROI}_{\text {source }}\right)-\text { mean }\left(\mathrm{ROI}_{\text {background }}\right)\right\} /\right. \\
& \text { standard deviation }\left(\mathrm{ROI}_{\text {background }}\right)
\end{aligned}
$$

This metric provides a second measure and is related to the detectability of the line source as a function of its activity concentration. For a given source geometry and activity, higher CNR implies better detectability.

\section{Effects of temperature}

Based on unanticipated trends in the intrinsic background, and evidence that these findings might be temperature-related, we investigated the relationship between temperature and background counting rate measurements for the clinical Biograph scanner. LSO light output and PMT gain are known to have temperature dependencies around room temperature. The magnitude of the dependence depends on the specific scintillation crystal sample and PMT type [13-18].

Biograph background acquisitions were performed with a 6 ns timing window and 350-650 keV energy window. Instantaneous singles measurements were recorded on a block-by-block basis over extended periods of time (up to $6 \mathrm{~h}$ ). Simultaneously, external scanner temperature was measured with a thermometer (DeltaTRAK Professional, Pleasanton, CA. Model No. 08J1) taped to the interior of the scanner bore and internal temperature was measured via sensors on the scanner electronic boards. Room temperature was modulated by a central air conditioning system and was not manipulated other than natural cycling of the system around its set point.

Single event rates were recorded and used for subsequent analysis, as they are much higher than prompt and random counting rates and thus have less statistical uncertainty. Using the Matlab Curve Fitting Toolbox, linear least-squares regression was performed on singles data to evaluate the relationship between temperature and counting rate measurements.

\section{Results}

The intrinsic counting backgrounds (prompts and randoms) measured in the two scanners (phantom present, but containing no activity) are shown in Table 2. For the Biograph scanner, measurements at different lower energy thresholds show the effect of energy windowing on the background due to natural ${ }^{176} \mathrm{Lu}$ in the scintillator material. The majority of the detected events are random coincidences. The background in the Discovery-ST scanner is negligible and almost all events are true coincidences with very few randoms. The small number of detected coincidence events may come from high-energy cosmic rays passing through the detector ring and interacting twice.

For the Biograph scanner, the scatter fraction used in the NECR calculation was measured as described using high-statistics scans. Scatter fraction values were $0.43,0.40$, and 0.34 for lower-level energy thresholds of 350,375 , and $425 \mathrm{keV}$, respectively. For the Discovery-ST scanner a literature value of 0.45 for the scatter fraction in the 375-650 keV energy range was used [3].

Figure 1 shows decay series counting rate measurements for the 375-650 keV energy window over a line source activity range of approximately $2-100 \mathrm{kBq} / \mathrm{ml}$. The intrinsic background rates from Table 2, which were acquired at a different time, also are shown.

\section{Table 2}

Intrinsic measured prompt and random counting rates (per second) with no activity in the scanner field-of-view. A dash indicates that the corresponding measurement was not taken.

\begin{tabular}{llllll}
\hline Energy Window & \multicolumn{2}{l}{ Siemens Biograph Reveal 16 } & & \multicolumn{2}{l}{ GE Discovery-ST } \\
\cline { 2 - 3 } \cline { 6 - 7 } \cline { 6 - 7 } & $\begin{array}{l}\text { Prompts } \\
\left(\mathrm{s}^{-1}\right)\end{array}$ & $\begin{array}{l}\text { Randoms } \\
\left(\mathrm{s}^{-1}\right)\end{array}$ & & $\begin{array}{l}\text { Prompts } \\
\left(\mathrm{s}^{-1}\right)\end{array}$ & $\begin{array}{l}\text { Randoms } \\
\left(\mathrm{s}^{-1}\right)\end{array}$ \\
\hline $350-650 \mathrm{keV}$ & 375 & 346 & & - & - \\
$375-650 \mathrm{keV}$ & 361 & 349 & & 0.29 & 0.007 \\
$425-650 \mathrm{keV}$ & 280 & 277 & & - & - \\
\hline
\end{tabular}


(a)
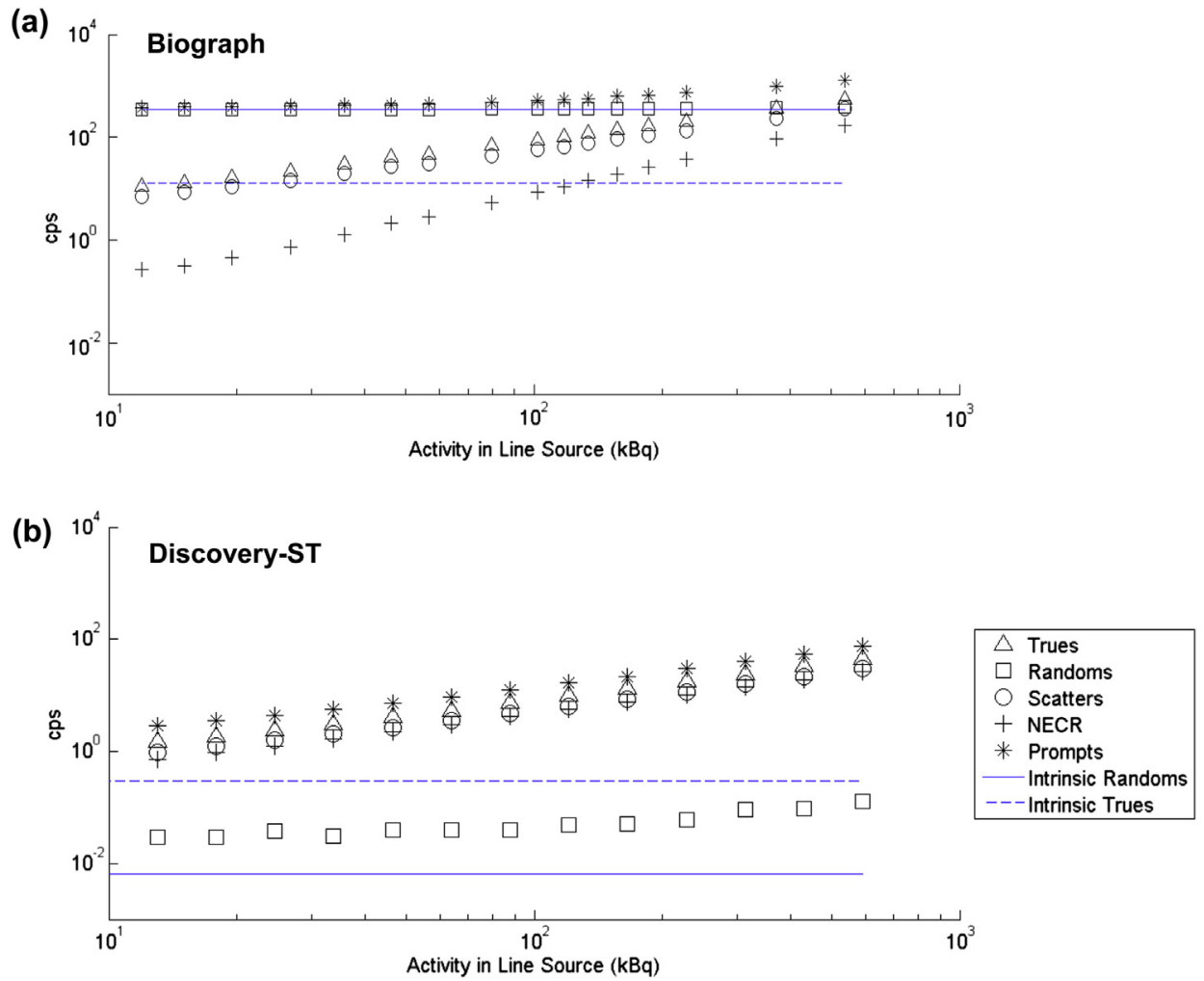

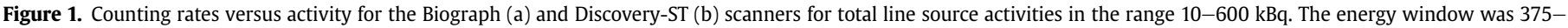

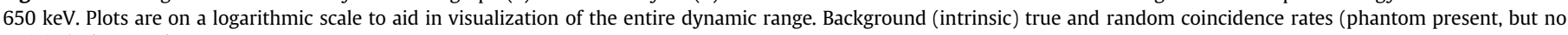
activity) also are shown.

The plots are shown on a logarithmic scale to improve visualization over the large dynamic range of the measurements, especially for the lower activity measurements that are the focus of this study.

Figure 2 is a comparison of the Biograph and Discovery-ST NECR curves up to an activity of $100 \mathrm{kBq} / \mathrm{mL}$, and sinogram CNR values up to an activity of $40 \mathrm{kBq} / \mathrm{mL}$. All data correspond to an energy window of 375-650 keV. The Discovery-ST scanner consistently had higher NECR values and CNR than the Biograph scanner, and the large difference in these values at low activities is primarily due to the effects of the higher intrinsic background present in the Biograph scanner. At the regular activities used in clinical studies, differences in NECR values between the two scanners are less pronounced.

Figure 3 illustrates the NECR curves and CNR results for the Biograph scanner as the lower energy threshold is varied. There is a trend towards slightly better CNR performance at higher energy thresholds. However, because the performance is dominated by the background ${ }^{176} \mathrm{Lu}$ signal, and since this background is not a strong function of the energy threshold over this range of practical lower energy threshold settings (see Table 2), the effects were modest.

At the lowest investigated activities, we noted that the rate of true coincidences measured on the Biograph scanner was sometimes lower than the average measured background (intrinsic) trues rate for the scanner (Fig. 1). Random statistical fluctuations were ruled out as a cause. One possible explanation for this observation could be small shifts in the measured background rate over time. Because the background events dominate the measurements at the lowest activity levels studied, even small effects can significantly impact data collected in this low-activity regime, whereas they may be quite insignificant when larger activities are present in the field of view. One plausible cause for shifts in the background event rate could be changes in temperature, as both LSO light output and PMT gain are known to have temperature dependencies and background events have a broad energy distribution that make the counting rate within an energy window susceptible to changes in the signal amplitudes. As described in the methods section this prompted further studies to look for correlations between temperature and counting rates.

Figure 4 illustrates the observed relationship between temperature and intrinsic singles rate for two separate long acquisitions obtained on the Biograph scanner. There was a tendency for temperature to steadily increase by $0.5-0.6{ }^{\circ} \mathrm{C}$ over a multi-hour acquisition time. Furthermore, when the singles rate was plotted as a function of temperature, there was a clear correlation. The $R^{2}$ values for linear-least squares regression were 0.78 and 0.74 for the two acquisitions. The results presented here are for external temperature measurements that had a precision of $0.05{ }^{\circ} \mathrm{C}$. On-board temperature measurements (not shown) were of poorer precision, $0.1{ }^{\circ} \mathrm{C}$, which was insufficient given the small range over which temperature fluctuated during the acquisition time. Nonetheless, we found that on-board temperature measurements were consistent with external (interior of scanner bore) temperature measurements.

A further interesting and possibly related observation occurred when recording counting rates immediately following a CT scan on the Biograph PET/CT scanner. Figure 5a shows the observed intrinsic singles rate for another long acquisition during which two CT scans were performed. The data shows there are discontinuities immediately after the CT scan is performed, when temperature (as recorded by the DeltaTrak thermometer attached to the scanner bore) and the recorded singles event rate decrease significantly. Following a CT scan, the observed singles rate was best fit with an 

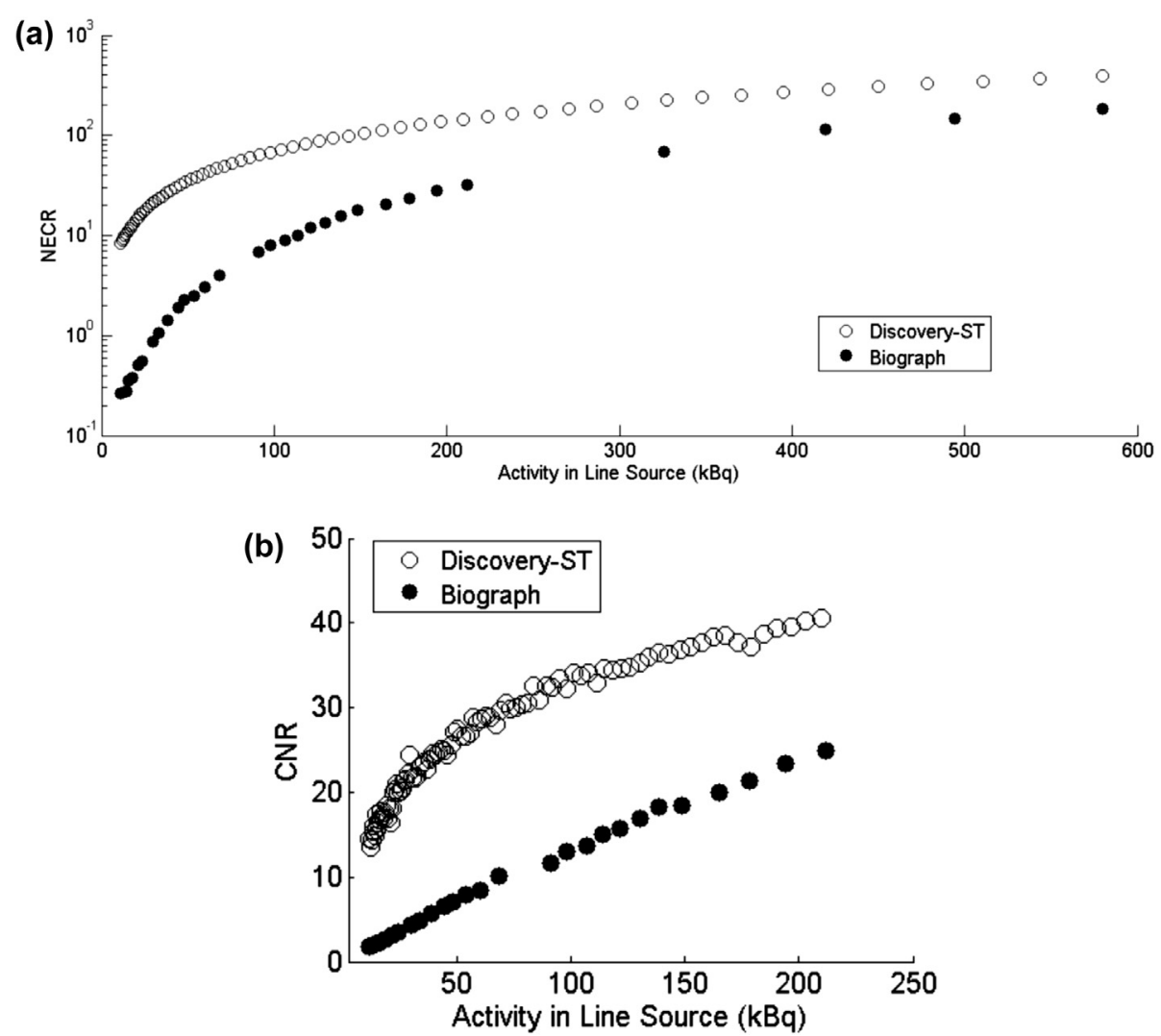

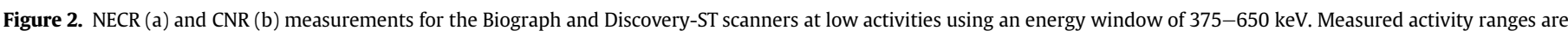

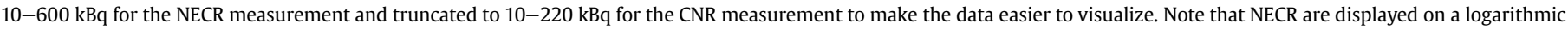
scale.

exponential function with time constants in the range of $6-8 \mathrm{~min}$ ( $R^{2}$ values 0.89 and 0.90 for the two datasets). Closer inspection of singles data revealed that the strong trends in Fig. 5a could be attributed almost entirely to the PET detectors in the ring closest to the CT scanner. The change in singles counting rate coincided with a small drop in the measured temperature that occurred immediately after CT scanning (Fig. 5b), and once again the singles rate was strongly correlated with temperature (Fig. 5c), and with $R^{2}$ values of 0.81 and 0.80 for the data acquired following each CT scan. These trends suggest that operation of the CT scanner component on the Biograph scanner may perturb the PET system temperature such that background counting rate measurements are transiently affected for tens of minutes afterwards. Another possible explanation for this effect (either alone or in combination with temperature related effects) would be LSO phosphorescence [18,19], a slow decay component caused by the population of traps in the scintillator by the high flux of scattered X-rays from the CT scanner. This slowly decaying component could create a large number of low-amplitude pulses that trigger the discriminators. Since these are below the energy threshold, they are not recorded, but they will increase dead time and thus may reduce the observed singles rate.

In contrast to these results, no correlation between temperature and counting rate was observed for measurements taken on the Discovery-ST scanner.

\section{Discussion}

Biograph scatter fraction and intrinsic counting rate measurements were consistent with previous investigations with the same PET scanner $[4,9]$. Intrinsic randoms are primarily due to two $\beta^{-}$ particles from separate ${ }^{176} \mathrm{Lu}$ decays, while intrinsic trues are primarily due to detection of a $\beta^{-}$particle in coincidence with a $\gamma$-ray from the same ${ }^{176}$ Lu decay [11]. Figure 2 indicates that the intrinsic randoms rate on the Biograph scanner was much higher than the intrinsic trues rate. The high randoms rate can be attributed to the nearly $100 \%$ efficiency of LSO for stopping ${ }^{176} \mathrm{Lu} \beta^{-}$particles, which deposit their energy in the same crystal in which they were emitted. $\gamma$-rays travel much longer distances than $\beta^{-}$particles, and may or may not interact in the Biograph's LSO detector ring, resulting in a lower detection efficiency for intrinsic true coincidences.

Intrinsic prompt counting rates were approximately 1000 times lower for the Discovery-ST scanner than the Biograph scanner. Most of the prompt events in the Biograph scanner are randoms. Decays contributing to Discovery-ST background are presumed to have originated externally to the scanner, since BGO does not contain any significant radioactive isotopes. External sources present in the scanner's vicinity included a nearby ${ }^{82} \mathrm{Rb}$ generator and a rodshaped ${ }^{68} \mathrm{Ge}$ normalization source located at the foot of the scanner bed. However, given the energy window used, and the prevalence of true coincidences over random coincidences, a very likely source for the small number of background events is cosmic radiation.

Decay series acquisitions showed that the Discovery-ST had significantly better NECR performance and sinogram CNR across the entire activity range studied when compared with the Biograph scanner. The primary cause of the difference at these activity levels is the effect of the ${ }^{176} \mathrm{Lu}$ background counting rates on both these metrics. The Biograph scanner consists of $\sim 9585$ cc of LSO material, corresponding to an activity of $\sim 3.1 \mathrm{MBq}$ of ${ }^{176} \mathrm{Lu}$. In contrast, the 
(a)

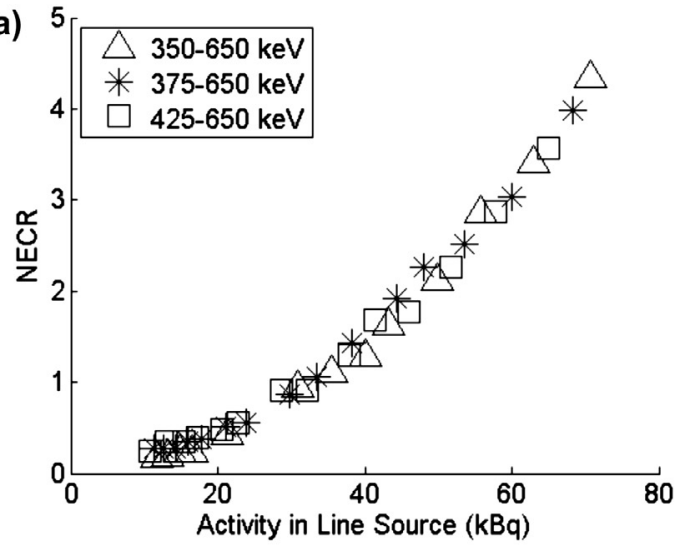

(b)

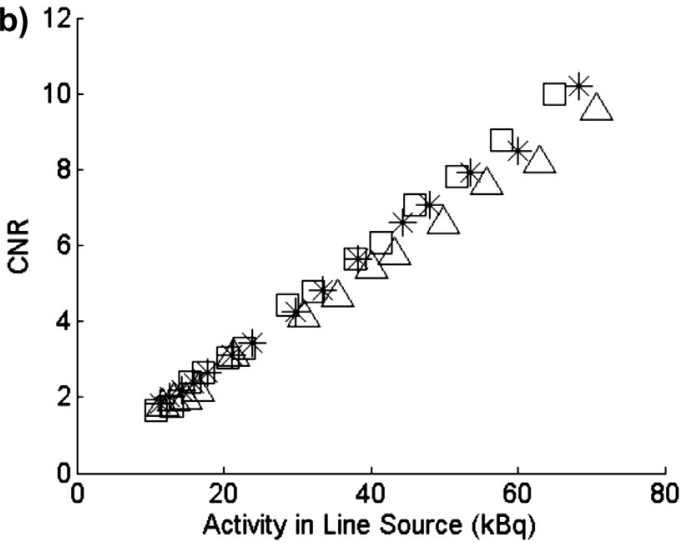

Figure 3. (a) Comparison of NECR and (b) CNR for lower energy window settings of 350,375 , and $425 \mathrm{keV}$ for the Biograph scanner. Measured activity range is $10-70 \mathrm{kBq}$.

maximum source activity introduced in this study was $600 \mathrm{kBq}$, of which only $\sim 138 \mathrm{kBq}$ (70 cm line source, $16.2 \mathrm{~cm}$ axial field of view) was actually within the axial field of view. A second likely cause is the somewhat higher sensitivity of the Discovery scanner (see Table 1) which is largely due to the higher stopping power of BGO and the slightly longer crystals used in this scanner. This is partially offset by the somewhat shorter axial field-of-view and larger ring diameter which reduces solid angle coverage compared to the Biograph scanner.

There was a trend towards improving performance with higher energy thresholds on the Biograph scanner, but the differences were small. Although increasing the lower-energy threshold will somewhat reduce the contributions of background events (see Table 2), it will also eliminate detection of some true coincidence events from the source. We conclude that lower energy thresholds above $350 \mathrm{keV}$ do not significantly reduce the deleterious effect of ${ }^{176}$ Lu background counts for low-activity studies.

At the very lowest activity levels studied, we noted in some experimental runs that the Biograph coincidence rates were consistently below the previously measured intrinsic background counting rates, even though there was a low-activity source present. We hypothesized this might be a temperature effect, as both LSO light output and PMT gain have a temperature dependence which would lead to temperature dependent pulse amplitudes, thus changing the assumed relationship between pulse amplitude and the energy deposited in the detector, and therefore the number of events falling within the energy window. Indirect evidence for this came from the fact that the intrinsic coincidence rate we reported was an average over a 12-h overnight acquisition, while our decay series measurements reflect an average over individual 5-25 min
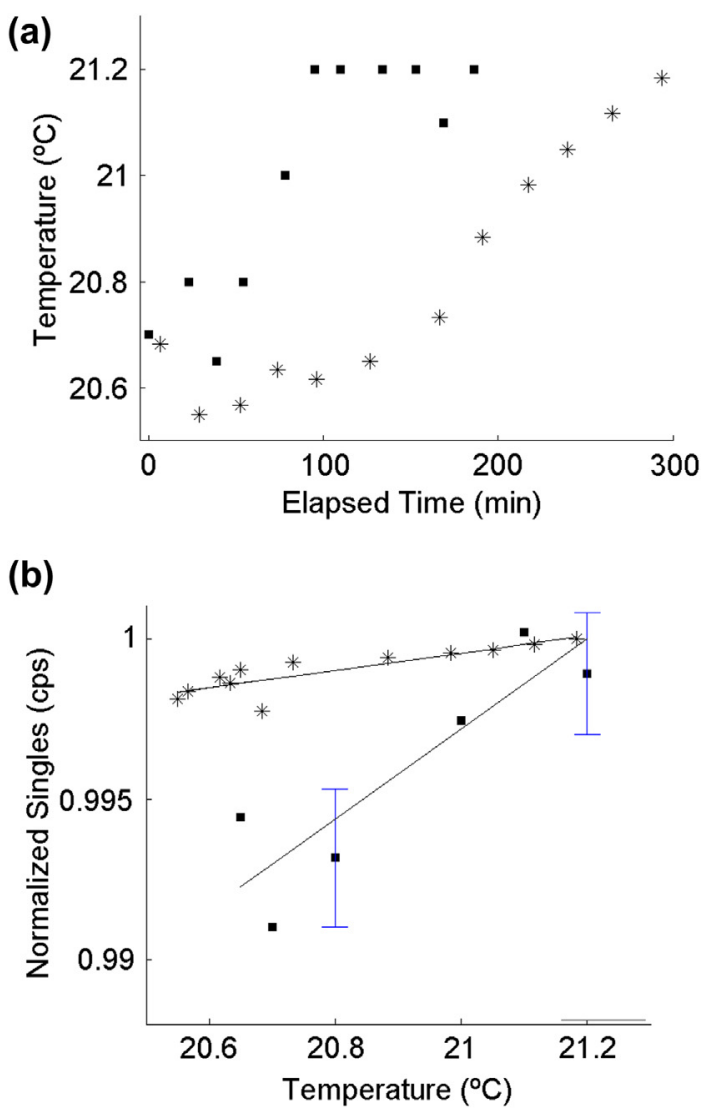

Figure 4. (a) Change in temperature versus elapsed time for two different multi-hour acquisitions on the Siemens Biograph scanner (each acquisition denoted by a different symbol). (b) Recorded singles rate due to LSO background (normalized to arbitrary units) versus temperature for the same two acquisitions. Solid lines represent linear fit, with $R^{2}$ values of 0.78 and 0.74 . For the case of multiple counting rate measurements at a single temperature, sample mean and error bars are shown. Error bars are +1 - one standard deviation of the mean counting rate.

frames acquired in the early evening, and, in general, facility temperature was observed to be lower at night. To study this more systematically, further decay series were acquired while monitoring scanner temperature. A strong linear correlation was found between the intrinsic background singles counting rate and temperature. We also noted that small temperature changes occurred immediately after a CT scan was performed. This was correlated with an abrupt change in the measured singles rates with a recovery time characterized by an exponential with a time constant of 6$8 \mathrm{~min}$. Another possible cause for the observed change in counting rate following a CT scan is X-ray induced phosphorescence in the LSO crystals.

In all cases studied, the changes in singles rates with bore temperature and following CT operation were small. However, because the dominant source of events at the low activities studied are random coincidences from ${ }^{176} \mathrm{Lu}$ background singles, the effects on NECR may still be significant. At the much higher activities used for clinical studies, it is not expected that these temperature effects would manifest themselves, as the background is negligible compared to events from the source, and the fraction of $511 \mathrm{keV}$ photopeak counts within the energy window are relatively immune to very small temperature-related shifts in the pulse amplitude.

In conclusion, these studies focused on the NECR performance of two commercial clinical PET/CT platforms in the unexplored regime of very low activity concentrations that might be encountered in emerging applications such as imaging radiolabeled cell populations and nanoparticles, or at very late time points in dynamic scans of 

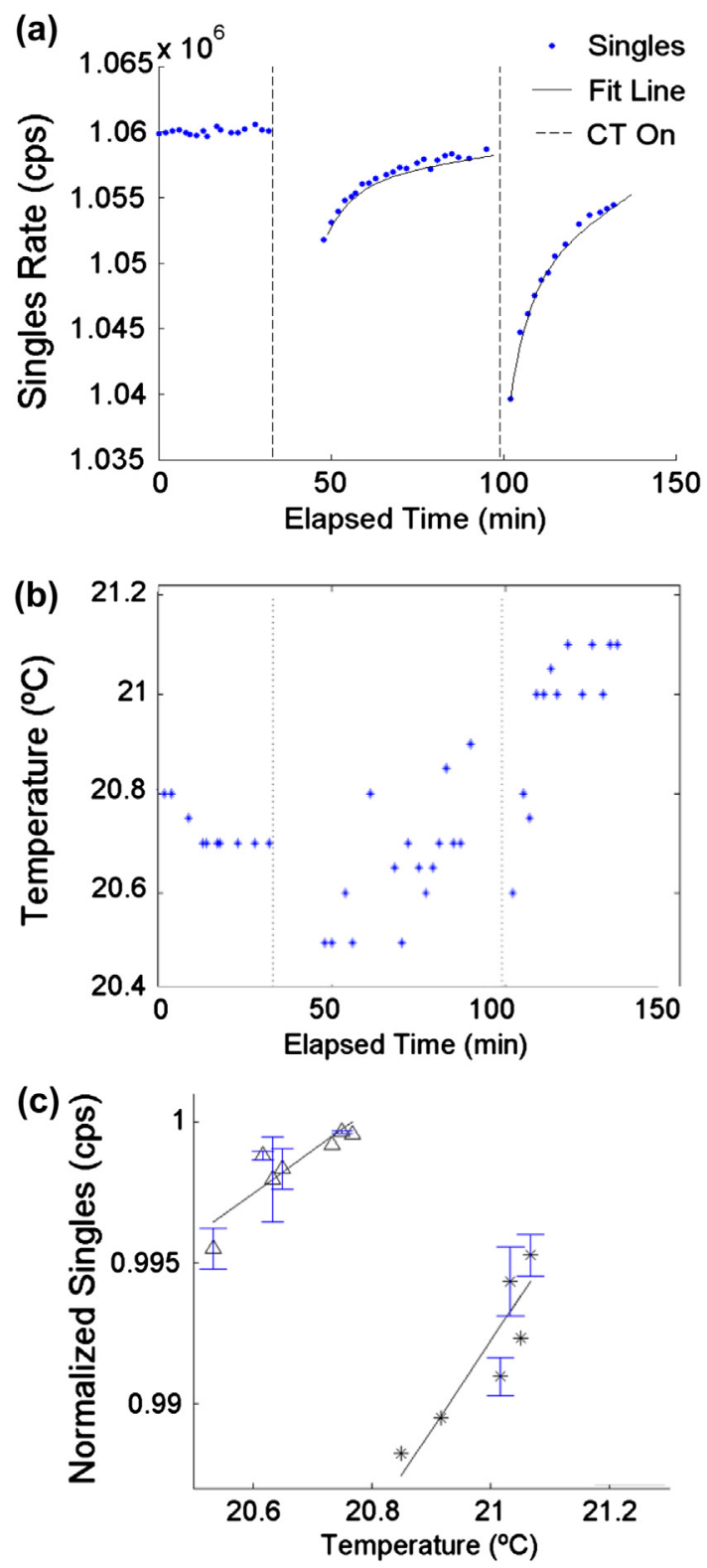

Figure 5. Singles rate versus elapsed scan time for an acquisition during which two CT scans also were acquired. Exponential fits are also shown. (b) Temperature measurements recorded during the acquisition. Vertical lines in both (a) and (b) indicate CT operation. (c) Temperature plotted versus normalized singles rate for same data. The two different symbols correspond to the data obtained following the two different CT scans. Measured $R^{2}$ values are 0.81 and 0.80 , respectively. Where multiple counting rate measurements were obtained for a single temperature, sample mean and error bars (+l- one standard deviation) are shown.

short half-life radionuclides. In this regime, we conclude that the background from ${ }^{176} \mathrm{Lu}$ significantly reduces NECR and that the variability with temperature of the intrinsic background upon which the faint signal sits, leads to complications that will make correction for this background challenging. For studies at these very low activities ( $<1 \mathrm{MBq}$ ), we therefore recommend the use of a BGO-based PET scanner where the background is negligible and the NECR performance much higher compared with an LSO-based system that has a similar detector and scanner geometry.

\section{Acknowledgments}

The authors wish to thank Bruce Finley and Ziad Abumughaiseeb for technical assistance and Drs. Abhijit Chaudhari, Gregory Mitchell, Rosalie Hagge, Yongfeng Yang, Jinyi Qi, Michel Tohme, Steven Falen and C. Chang Lee for useful discussions. This work was supported by the National Science Foundation Graduate Research Fellowship Program, the UC Davis Molecular Imaging Training Grant NIH T32 EB003827, and California Institute for Regenerative Medicine (CIRM) Tools and Technologies Grant \#RT1-01019.

\section{References}

[1] National Electrical Manufacturers Association. NEMA standards Publication NU 2-2007. Performance measurements of positron emission tomographs. Rosslyn, VA: National Electrical Manufacturers Association; 2007.

[2] Watson CC, Casey ME, Eriksson L, Mulnix T, Adams D, Bendriem B. NEMA NU 2 performance tests for scanners with intrinsic radioactivity. J Nucl Med 2004;45:822-6.

[3] Bettinardi V, Danna M, Savi A, Lecchi M, Castiglioni I, Gilardi MC, et al. Performance evaluation of the new whole-body PET/CT scanner: Discovery-ST. Eur J Nucl Med Mol Imaging 2004;31:867-81.

[4] Eckardt J, Herzog H, Scafers KP, Kapplinger S, Schober O. Impact of lower energy threshold on the NEMA NU2-2001 count-rate performance of a LSO based PET-CT. Scanner Nuklearmedizin 2008;47:210-4.

[5] Martinez MJ, Bercier Y, Schwaiger M, Ziegler SI. PET/CT biograph sensation 16 performance improvements using faster electronics. Nuklearmedizin 2006;45:126-33.

[6] Mawlawi O, Podoloff DA, Kohlmyer S, Williams JJ, Stearns CW, Cupl RF, et al. Performance characteristics of a newly developed PET/CT scanner using NEMA standards in 2D and 3D modes. J Nucl Med 2004;45:1734-42.

[7] Biograph 2/biograph 6/biograph 16 system specifications. Siemens Medical Solutions USA, Inc; 2006.

[8] McIntosh B, Goertzen AL. Validation of GATE simulations of the ${ }^{176} \mathrm{Lu}$ intrinsic activity in LSO detectors, 2009 IEEE nuclear science symposium-medical imaging conference record. Orlando, FL: IEEE; 2009. p. 3426-8.

[9] Yamamoto S, Horii H, Hurutani M, Matsumoto K, Senda M. Investigation of single, random, and true counts from natural radioactivity in LSO-based clinical PET. Ann Nucl Med 2005;19:109-14.

[10] Huber JS, Moses WW, Jones WF, Watson CC. Effect of 176-Lu background on singles transmission for LSO-based PET cameras. Phys Med Biol 2002;47: 3535-41.

[11] Goertzen AL, Suk JY, Thompson CJ. Imaging of weak-source distributions in LSO-based small-animal PET scanners. J Nucl Med 2007;48:1692-8.

[12] Melcher CL. Scintillation crystals for PET. J Nucl Med 2000;41:1051-5.

[13] Gervino G, Monticone E. Characterization and performance of BGO crystals for positron emission tomography. Sens Actuators 1994;42:487-90.

[14] Melcher CL. Lutetium orthosilicate single crystal scintillator detector US patent $5025151 ; 1991$.

[15] Naud JD, Tombrello TA, Melcher CL, Schweitzer JS. The role of cerium sites in the scintillation mechanism of LSO. IEEE Trans Nucl Sci 1996;43:1324-8.

[16] Photonis. Photomultiplier tubes: principles and applications 2002.

[17] Suzuki H, Tombrello TA, Melcher CL, Schweitzer JS. Light-emission mechanism of $\mathrm{Lu}_{2}\left(\mathrm{SiO}_{4}\right) \mathrm{O}-\mathrm{Ce}$. IEEE Trans Nucl Sci 1993;40:380-3.

[18] Yang K, Melcher CL, Rack PD, Eriksson LA. Effects of calcium codoping on charge traps in LSO: Ce crystals. IEEE Trans Nucl Sci 2009a;56:2960-5.

[19] Mao R, Zhang L, Zhu RY. Gamma Ray Induced Radiation Damage in PWO and LSO/LYSO Crystals. In: Nuclear science symposium conference record (NSS/ MIC), 2045-2049; 2009. 